\title{
INDICADORES PARA EVALUAR PROGRAMAS DE ORIENTACIÓN EDUCATIVA
}

\author{
INDICATORS TO EVALUATE EDUCATIONAL GUIDANCE PROGRAMS
}

\author{
Nuria Manzano* \\ Centro de Orientación, Información y Empleo (COIE) \\ Universidad Nacional de Educación a Distancia (UNED)
}

\section{RESUMEN}

Este artículo expone el proceso y resultados de la investigación llevada a cabo, relativa a la elaboración de un sistema de indicadores para evaluar programas de orientación educativa. Dicho sistema consta de seis factores de calidad, veinte dimensiones y cien indicadores. Una vez realizado, se validó su contenido mediante un juicio de expertos y a través de la técnica Delphi. Como resultado, se mejoró la formulación y ubicación de los indicadores, la medida más adecuada y se obtuvo una ponderación de cada uno de ellos.

Palabras clave: Evaluación de programas de orientación, Indicadores de ejecución, Programas comprensivos de orientación.

\begin{abstract}
This article presents the process and results of a research which, focused on the development of indicator systems to evaluate guidance programs. Such system has six quality factors, twenty dimensions and one hundred indicators. Afterwards, content validation was carried out through experts judgement (Delphi technique). Results allowed to improve definition and location of indicators, to get the most appropriate measure and a ponderation of each indicator.
\end{abstract}

Key words: Counseling evaluation program, Performance indicators, Comprehensive guidance program.

\section{Introducción}

La evaluación de programas de orientación está siendo objeto de atención desde los años setenta y, especialmente, en países donde existía ya una tradición en labores de orientación educativa. Estados Unidos ha sido uno de los países que comenzó a reflexionar sobre

\footnotetext{
* Doctora en Pedagogía. Orientadora en el COIE de la UNED. Realizó la tesis doctoral sobre el tema «Elaboración y validación de un Sistema de Indicadores para evaluar programas de orientación educativa». Sigue trabajando en temas de evaluación de programas y elaboración de indicadores.
} 
la necesidad de elaborar sistemas para evaluar los efectos de las intervenciones orientadoras, debido a los recortes presupuestarios que se habían hecho en servicios sociales $\mathrm{y}$, por tanto, a la importancia de que los profesionales dieran cuenta de la efectividad de sus programas. Sin embargo, de la necesidad teórica a la aplicación práctica y, más aún, a la disponibilidad de instrumentos válidos y fiables que pudieran responder a esta prioridad, quedaba mucho camino por recorrer. En ese camino han existido algunos problemas o dificultades que han retrasado el indispensable avance en este campo.

Hasta ahora la mayoría de los estudios hechos en nuestro país se han centrado, según Moreno Castellano y otros (1993) en:

a) Autoevaluaciones a pequeña escala en distintos centros;

b) Examen de las actitudes de los estudiantes sobre la orientación más que la evaluación de sus efectos;

c) Expectativas hacia la orientación, tanto de profesores como de alumnos.

Sin embargo, la evaluación de la eficacia de la orientación en España queda aún pendiente. Sanz Oro (1996) destaca la necesidad de la evaluación como componente imprescindible en el diseño de todo programa y expone una serie de razones — que se completan aquí- que justifican la necesidad de evaluar los programas de orientación, a partir del análisis de los trabajos de varios autores (Breakwell, 1987; Delworth y Hanson, 1980; De Miguel, 1985; Gade, 1981; Hayden y Pohlmann, 1981; Matthay, 1988; Shaffer y Atkinson, 1983; Tejedor, 1990; Wheeler y Loesch, 1981; Wilson, 1985) (ver tabla 1).

En definitiva, el propósito preferente de la evaluación será identificar la efectividad de la ayuda dada para poder señalar las mejoras necesarias. Esto implica establecer una serie de criterios conducentes al éxito, que puedan evaluarse a corto, medio y largo plazo. Estos criterios llevan, según Krumboltz (1974), a la promulgación de objetivos de conducta, a la identificación de objetivos específicos para cada orientado, y a una evaluación válida de los resultados.

Esta necesidad de evaluar debe concretarse en una serie de objetivos de evaluación. La mayoría de los autores (Pérez Juste, 1992; Tejedor, 1994; Rodríguez Espinar y otros, 1993) concuerdan en destacar que los propósitos o finalidades de la evaluación en la orientación son:

a) Emitir un juicio sobre el valor y la utilidad de un programa;

b) Asistir a quienes toman las decisiones sobre la implantación, ampliación o suspensión de programas, $\mathrm{y}$

c) Contribuir a la mejora de los programas.

Entre las diversas razones que se apuntan para comprender esta situación, hay una fundamental, según Loesch (1981) y Schaffer y Atkinson (1983): la escasa formación que reciben los orientadores en cuestiones de evaluación. Otros autores, en cambio, dejan en un segundo plano la importancia de la formación porque consideran que es una justificación que encubre otras razones de los orientadores para no afrontar este reto. Frith y Clark (1982) afirman que la realidad es que los orientadores tienen una percepción equivocada de lo que significa «evaluar la orientación». La perciben como una herramienta propia y típica de los investigadores, excesivamente complicada y costosa para ellos. Lo que refleja esta actitud es la existencia de una serie de mitos en la profesión que, según él, conviene eliminar para 


\section{TABLA 1: Razones de la necesidad de evaluar la orientación.}

- Recibir un constante feedback sobre el grado de efectividad del programa de orientación, a través de unos datos recogidos sistemáticamente

- Comprobar si el proceso de orientación está siendo satisfactorio para los destinatarios del mismo y está respondiendo a sus necesidades

- Elegir y utilizar las técnicas de orientación sobre la base de su efectividad

- Poder justificar la eliminación de actividades y recursos de orientación inútiles

- Mejora progresiva del programa, pues los datos obtenidos sirven para desarrollar nuevos servicios o adaptar los ya existentes con el objetivo de satisfacer los objetivos del programa

- Tener una base fundamentada y justificada, lejos de intuiciones y sesgos, sobre la que sustentar las decisiones que se adopten respecto al programa

- Informar a la comunidad educativa respecto de las intervenciones realizadas. Así, padres, profesores y alumnos, según esta información, definirán mejor las necesidades y metas del programa, a la vez que pueden proporcionar mayor apoyo material y personal para su continua mejora. En definitiva, favorecer su participación y una toma de decisiones más fiable.

- Controlar la calidad de la orientación en el centro

- Preparar a los estudiantes en una serie de competencias básicas

- Facilitar la evaluación del profesorado y el orientador con el objeto de favorecer el cambio educativo

- Para ayudar a compensar las desigualdades educativas, al evaluar el funcionamiento de los departamentos de orientación en entornos socioculturales diferentes.

- Introducir criterios de racionalidad en el proceso. Según Tejedor (1990) «así ha surgido en los ámbitos sociales y educativos la preocupación por el diagnóstico y evaluación de las actuaciones educativas» $\mathrm{y}$, por lo tanto, también de las orientadoras.

- Buscar indicadores para valorar los programas, los procesos y los resultados de la función orientadora (De Miguel, 1985). De aquí se desprende que el orientador necesitará realizar la evaluación del programa como medio para obtener elementos de juicio en los que apoyar su actividad o labor profesional.

que el criterio que guíe el programa sean las necesidades de los alumnos, no estos mitos que a continuación se enumeran:

a) Se necesitan unas habilidades específicas para llevar a cabo la evaluación;

b) Son demasiado caras;

c) Es difícil obtener datos de los alumnos porque no son buenas fuentes de información;

d) Los datos deben obtenerse sólo a través de procedimientos objetivos, no subjetivos;

e) No es útil recoger datos de evaluación de los padres;

f) La evaluación requiere mucho tiempo;

g) La evaluación necesita una amplia muestra de participantes;

h) Los directores y la administración no apoyan la evaluación;

i) La evaluación se hace principalmente para intereses de investigación y publicación; 
j) La interpretación de los datos de evaluación necesita análisis estadísticos muy amplios.

En definitiva, es importante entender que la evaluación ya no es un instrumento al servicio de los gobiernos en períodos de crisis para reducir presupuestos sino el eje, junto a la planificación, que permite mejorar la intervención/resultados y el argumento para demostrar la utilidad y necesidad de la orientación educativa.

El trabajo que se presenta a continuación es la conclusión de una revisión de los sistemas de evaluación de la orientación que existen actualmente. Lo que motivó emprender esta investigación, con los condicionantes que supone la escasa investigación al respecto y la reducida experiencia práctica, fue:

a) la ausencia de propuestas metodológicas que faciliten la práctica evaluadora de la orientación educativa,

b) la apreciación no contrastada de una escasa cultura de evaluación entre los orientadores, $\mathrm{y}$,

c) las necesidades detectadas en los orientadores.

Pretendíamos elaborar una metodología - Sistema de Indicadores - que permitiera responder objetiva y eficazmente a esta cuestión. Sin embargo, antes de ofrecer este instrumento para su aplicación era necesario validar su contenido y afinar su diseño y estructura.

\section{Objetivos}

Los objetivos que guiaron la investigación fueron los siguientes:

1. Elaborar un sistema de indicadores de evaluación que permita conocer y analizar el estado actual de la orientación educativa y, en último término, tomar decisiones de mejora.

- Elaborar indicadores de calidad de la orientación. Tales indicadores han de responder a criterios de calidad educativa y según los requisitos de constructo, medida y formalización.

- Formalización de dichos indicadores en un Sistema, de manera que los diferentes factores, dimensiones e indicadores de orientación estén relacionados.

- Facilitar un procedimiento y un sistema de evaluación participativa, tanto de evaluación interna como externa

2. Validar el S.I. a través de un juicio de expertos

- Mejorar la definición y claridad de cada indicador

- Seleccionar los indicadores más importantes y obtener una ponderación de cada uno de ellos

- Obtener el tipo de medida más adecuada para cada indicador

- Mejorar la estructura del sistema y ubicación de cada indicador

El proceso llevado a cabo fue:

a) Elaboración del Sistema de Indicadores (S.I.);

b) Validación de contenido del S.I. mediante un juicio de expertos; 
c) Análisis de los resultados obtenidos, y;

d) Revisión del S.I. con las aportaciones recogidas.

\section{Metodología o Procedimiento}

\section{Elaboración del Sistema de Indicadores}

La evaluación continua de un programa de orientación ejerce un papel fundamental en la mejora del programa. Se diseña paralelamente a éste, de manera que se pueda obtener información de todas las partes del programa. Sólo así es posible detectar la funcionalidad, eficiencia y eficacia de los programas, y no centrar la evaluación sólo en los resultados.

El sistema de indicadores, como instrumento de evaluación, se concibe como una fórmula de valoración y planificación de programas de orientación educativa que permite analizar la situación actual que tiene la orientación —en diferentes factores - , definir la situación deseable y planificar cómo vencer esa discrepancia. Su pretensión es responder a una doble demanda:

a) satisfacer tanto las exigencias de autocontrol que se suscitan dentro del departamento o equipo de orientación, $y$,

b) satisfacer las demandas promovidas por audiencias externas. En cualquier caso, el objetivo es obtener información útil para tomar decisiones que mejoren la calidad de la orientación, en cualquiera de sus aspectos tanto internos (mejorar el clima y la organización, trabajar con un programa adecuado y bien hecho, mejorar la intervención orientadora — adecuación de actividades y técnicas, seguimiento del nivel de respuesta de las actividades a los objetivos-, satisfacción del estudiante, logro de competencias, etc.), como externos (disponer de suficientes y adecuados recursos, adecuada y suficiente formación de los orientadores, apoyo legal y de asociaciones profesionales, etc.).

La utilidad de disponer de un sistema de evaluación continua de la orientación radica en que se dispone de información puntual y secuencial:

a) para conocer la efectividad del programa,

b) para tomar las decisiones necesarias que respondan mejor a las necesidades cambiantes de los alumnos y,

c) para mejorar el programa de orientación progresivamente.

Según Selden (1990), para elaborar indicadores deben seguirse tres fases:

a) organizar conceptualmente el modelo de indicadores;

b) seleccionar los indicadores dentro del modelo; $y$

c) desarrollo técnico de los indicadores, definiéndolos y haciéndolos operacionales para poder recogerlos. En el caso que nos ocupa, el proceso de elaboración del S.I. fue el siguiente (tabla 2).

Pues bien, después de revisar la literatura al respecto y analizar las diferentes definiciones existentes sobre calidad de la orientación educativa, se llegó a la conclusión de que el concepto de calidad es muy discutido. Muchos autores (Rodríguez Espinar, 1988a, 1988b, 


\section{TABLA 2: Proceso de elaboración de indicadores.}

\begin{tabular}{|c|c|}
\hline $\begin{array}{l}\text { Clarificación de la } \\
\text { evaluación }\end{array}$ & $\begin{array}{l}\text { 1. SENTIDO DE LA EVALUACIÓN DE LA ORIENTACióN } \\
\text { - Revisión de la literatura sobre el tema } \\
\text { - Especificación del concepto y calidad de la orientación } \\
\text { - Definición del modelo teórico subyacente, basado en los resultados de } \\
\text { investigación y el juicio de los expertos y audiencias implicadas, en el } \\
\text { que aparezcan explícitos los fenómenos implicados y las relaciones que } \\
\text { se establecen entre ellos. }\end{array}$ \\
\hline $\begin{array}{l}\text { Construcción del } \\
\text { sistema de } \\
\text { indicadores }\end{array}$ & $\begin{array}{l}\text { 2. ELABORACIÓN DE INDICADORES } \\
\text { - Identificación de los factores de calidad } \\
\text { - Especificación de dimensiones } \\
\text { - Formulación de indicadores, seleccionando un número limitado, } \\
\text { reduciendo su complejidad y siguiendo los requisitos de constructo, } \\
\text { medida y formalización. } \\
\text { 3. SínTESIS DEL SISTEMA DE INDICADORES } \\
\text { - Establecer relación entre indicadores } \\
\text { 4. REVISIÓN DE SISTEMA POR MEDIO DE JUICIO DE EXPERTOS }\end{array}$ \\
\hline $\begin{array}{l}\text { Procedimientos de } \\
\text { medida }\end{array}$ & $\begin{array}{l}\text { 5. ESTABLECER CRITERIOS DE EVALUACIÓN } \\
\text { 6. ESPECIFICAR LOS MEDIOS DE RECOGIDA DE INFORMACIÓN }\end{array}$ \\
\hline $\begin{array}{l}\text { Valoración según } \\
\text { requisitos de } \\
\text { indicadores }\end{array}$ & 7. ElabORACIÓN DE ESCALA PONDERADA DE VALORACIÓN \\
\hline
\end{tabular}

1991, 1993 y 1995; Lindsay, 1982; Goedegebuure y otros, 1990) presentan la calidad como un concepto multidimensional cuya valoración implica trabajar con varios criterios que están entremezclados. Sólo desde esta multidimensionalidad se pueden abordar algunas de las aproximaciones sobre las que se fundamenta el concepto de calidad, aún sabiendo que los resultados que disponemos no reflejan completamente y por sí solos la calidad de la orientación. Por lo tanto, la manifestación de la calidad de la orientación podría constatarse con la evidencia de que:

- Los objetivos y metas del programa de orientación reflejen el contexto en el que se desarrolla y, a su vez, los resultados respondan a las demandas de ese contexto.

- El programa de orientación pueda ponerse en práctica tal y como estaba previsto; y, de hecho, se ponga en práctica.

- El proceso de orientación explique y facilite la mayor productividad tanto del programa como del orientador. La relación que existe entre el proceso llevado a cabo (aplicación del programa de orientación y ejecución del orientador) y el producto obtenido

- Se consigan los resultados previstos con el menor coste en recursos, la mayor aplicación del programa y el menor esfuerzo y problemas en el proceso.

- Se consigan los objetivos previstos, independientemente de los medios empleados para ello y del proceso seguido. 
Después se estableció un modelo teórico subyacente - siguiendo el esquema Cipp de Stufflebeam - que ubica los factores de calidad, las dimensiones más importantes que componen cada factor y los indicadores que explicitan la información relevante en relación a la información disponible. La importancia de definir un modelo previo radica (Oakes, 1986) en que un S.I. es más que un conjunto de indicadores, ya que debe llevar implícita una concepción teórica sobre la organización, distribución y relación de las variables que lo configuran y un entramado de relaciones uni y/o bidireccionales entre ellas. Por otro lado, los ingredientes que debe seguir un modelo de efectividad escolar (Scheerens, 1991) son:

a) Un modelo de sistemas analíticos que identifique las variables de contexto, input, proceso y output;

b) Una red multinivel que separe e identifique las dimensiones de cada factor;

c) Perspectivas para ver las interrelaciones entre las variables definidas en diferentes niveles, en función de los resultados significativos que se encuentren en la investigación sobre la efectividad escolar;

d) Concreción aplicada de las interrelaciones (y, según esto, darles más o menos peso dentro del sistema de indicadores), que determinan la eficacia escolar, explicación causal y precisión futura.

Por tanto, es necesario construir este entramado que explique la efectividad de la orientación educativa. A continuación se representa de la siguiente forma (gráfico 1).

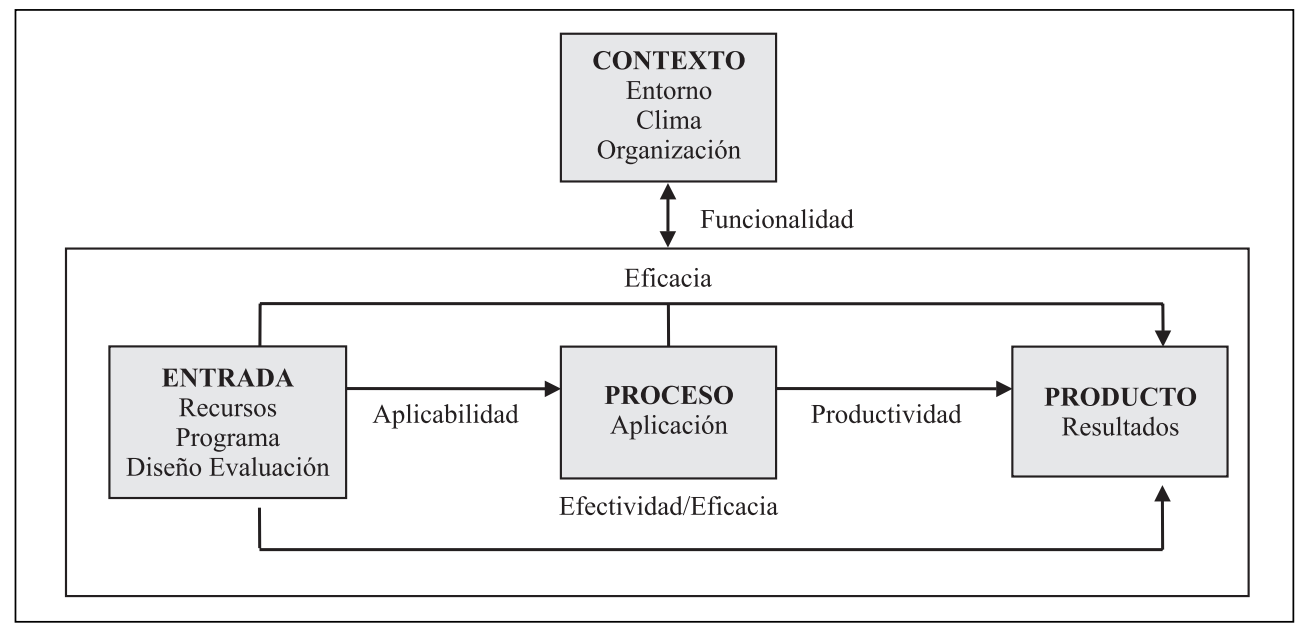

\section{GRÁFICO 1.}

Fundamentos evaluativos del sistema de indicadores.

Algunos ejemplos de cómo organizar la evaluación de la orientación a través de indicadores son los trabajos de Noman C. Gysbers y Drury \& Borders. En síntesis, el esquema de funcionamiento integra tanto los FACTORES,

- Contextuales (clima, organización, entorno): que permitan describir el contexto que condiciona y determina el desarrollo de la orientación. Estas variables tienen una 
incidencia en todas las demás, ayudan a interpretar y ponderar los resultados encontrados.

- De entrada (recursos humanos, materiales y políticos; diseño del programa; y, diseño de la evaluación): necesarias para conocer los apoyos con los que contamos, dónde se sitúan las fortalezas y problemas de la orientación; y necesarias también para planificar la acción orientadora.

- De proceso (aplicación del programa, ejecución del orientador): que se llevan a cabo para conocer el nivel de aplicación del programa y la calidad de la ejecución del orientador.

- De producto (múltiples resultados): los efectos obtenidos y el logro de objetivos previstos por el programa de orientación.

Como los CRITERIOS DE EVALUACIÓN:

- Funcionalidad: La coherencia entre el contexto (valores, expectativas y necesidades del entorno), los objetivos, metas y los resultados de la orientación. Es decir el grado en que los objetivos y metas del programa de orientación reflejan el contexto (valores, expectativas, necesidades, características del entorno) en el que se desarrolla; y, a su vez, los resultados responden a las demandas de ese contexto. Se produce así un círculo funcional.

- Eficiencia: La coherencia entre las entradas (recursos, programa), los procesos y los resultados de la orientación. Es decir, el grado en que se consiguen los resultados previstos con el menos coste en recursos, la mayor aplicación del programa y el menor esfuerzo y problemas en el proceso.

- Aplicabilidad: La coherencia entre las entradas (recursos, programa y diseño de la evaluación) y su aplicación. Es decir, el grado en que se puede poner en práctica aquello previsto.

- Productividad: La relación que existe entre el proceso llevado a cabo (aplicación del programa de orientación y ejecución del orientador) y el producto obtenido (1.productividad técnica del programa: resultados cuantitativos del programa; 2.productividad técnica del orientador: nivel de esfuerzo del orientador; 3.productividad de nuevos materiales).

- Eficacia / efectividad: La coherencia entre los objetivos y metas del programa y los resultados logrados. Es decir, el grado en que se consiguen los objetivos previstos, independientemente de los medios empleados para ello y del proceso seguido.

Pasamos ahora a la estructura del S.I. Está dividido en dos secciones:

- El Contenido de evaluación (gráfico 2): se compone de

1. Factores de calidad,

2. Dimensiones,

3. Indicadores.

Los factores son obligatorios y las dimensiones e indicadores combinan una parte obligatoria y una parte opcional, en función del contexto en donde se aplique.

- El Proceso de evaluación y mejora (tabla 3): se compone de cuatro partes relacionadas entre sí linealmente: 


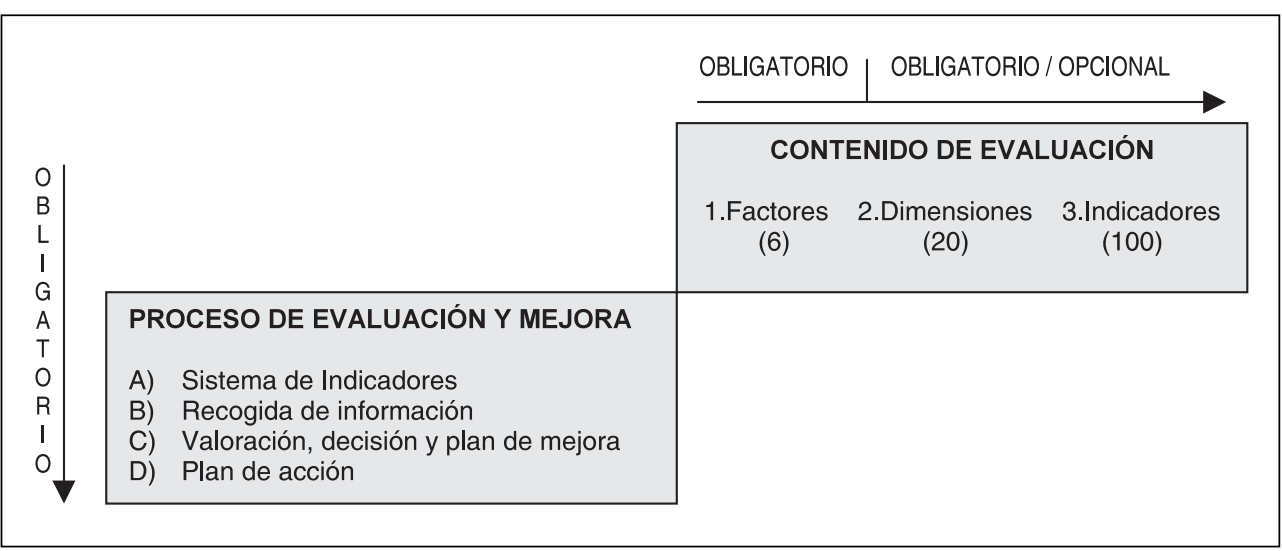

GRÁFICO 2.

Funcionamiento del Sistema de Indicadores.

TABLA 3: Estructura del Sistema de Indicadores.

A) SISTEMA DE INDICADORES

1. Factores de calidad

2. Dimensiones

3. Indicadores

B) RECOGIDA DE INFORMACIÓN

1. Agente evaluador

2. Método de evaluación

3. Instrumentos

4. Documentación

5. Audiencia

6. Contextualización

7. Temporalización

C) VALORACIÓN, DECISIÓN Y PLAN DE MEJORA

1. Valoración de indicadores y del programa

2. Relación de resultados de indicadores

3. Criterio y juicio valorativo

4. Tipo de decisión

5. Mejora de indicadores

D) PLAN DE ACCIÓN

1. Pauta para la concreción y aplicación de indicadores obligatorios
A) Sistema de Indicadores,
B) Recogida de información,
C) Valoración, decisión y plan de mejora, y
D) Plan de acción. Por tanto, constituye un proceso sistemático de evaluación y me- jora que no puede dividirse.


A partir de esta estructura, se pueden conocer las áreas de fortaleza y debilidad en el programa de orientación y en sujetos. Además, usado regularmente, puede ser una herramienta para mejorar la planificación y proceso de orientación educativa.

Se muestra a continuación el desglose de los 6 factores y las 20 dimensiones del sistema. Los 6 factores que definen la calidad de un programa de orientación educativa son (tabla 4).

TABLA 4: Factores de Calidad.
I. CONTEXTO
II. DISEÑO DEL PROGRAMA
III. RECURSOS
IV. APLICACIÓN DEL PROGRAMA
V. EVALUACIÓN DEL PROGRAMA
VI. RESULTADOS DEL PROGRAMA

La selección de dimensiones (20) se refiere a la concreción de los factores especificados previamente. El número de dimensiones por cada factor responde a las más significativas, no a todas las posibles (tabla 5).

Cada una de estas dimensiones tiene a su vez una serie de indicadores. Como ejemplo, se muestran a continuación los indicadores de la dimensión 5 «Diseño del programa comprensivo de orientación» (tabla 6).

\section{Validación del Sistema de Indicadores}

Sabemos que es una propuesta de evaluación parcial porque se detiene sólo en los aspectos más importantes implicados en la orientación y, por otro lado, esta evaluación se entronca en la misma evaluación del centro. Cada uno de estos elementos raramente son autónomos, por lo que la evaluación de programas de orientación está en relación con el cambio y/o mejora de otros elementos del centro educativo y con ciertas limitaciones, pues la evaluación del programa de orientación no implica la mejora del mismo en su totalidad.

La validación de contenido se realizó a través de la técnica Delphi con el objetivo de llegar a un consenso para extraer los indicadores más significativos y aplicables para evaluar la calidad de la orientación educativa. Dicha técnica consiste en el análisis de la opinión de varios expertos sobre un mismo tema. El proceso ha consistido en lo siguiente:

a) Se elaboró el cuestionario para recoger la información;

b) Se seleccionaron los grupos de expertos, según una serie de criterios;

c) Se aplicó el cuestionario a todos los expertos;

d) Se analizaron los datos, global y específicamente por indicadores, dimensiones y factores, así como por grupos de expertos;

e) Se revisó y modificó el sistema de indicadores con las respuestas y aportaciones recogidas. 


\section{TABLA 5: Dimensiones.}

\section{FACTOR I: CONTEXTO}

Dimensión 1. Características del entorno

Dimensión 2. Apoyo político y legal al programa comprensivo de orientación

Dimensión 3. Estructura del programa y organización del personal

Dimensión 4. Clima social en el que se desarrolla el programa

FACTOR II: DISEÑO DEL PROGRAMA

Dimensión 5. Diseño del programa comprensivo de orientación

\section{FACTOR III: RECURSOS}

Subfactor 1.1: Personales

Dimensión 6. Disponibilidad de personal

Dimensión 7. Cualificación inicial del personal

Dimensión 8. Competencia y ejecución del orientador (Supervisión)

Dimensión 9. Actualización y formación profesional continua (Desarrollo profesional)

Subfactor 1.2: Financieros

Dimensión 10. Presupuesto

Dimensión 11. Infraestructuras

Dimensión 12. Materiales y equipamiento

Subfactor 1.3: Políticos

Dimensión 13. Política/Legislación ministerial, autonómica, local y del centro

Dimensión 14. Estandares de las asociaciones profesionales de orientación y código ético

\section{FACTOR IV: APLICACIÓN DEL PROGRAMA}

Dimensión 15. Aplicación real del programa

\section{FACTOR V: EVALUACIÓN DEL PROGRAMA}

Dimensión 16. Diseño de la evaluación del programa comprensivo de orientación

\section{FACTOR VI: RESULTADOS DEL PROGRAMA}

Dimensión 17. Evaluación de resultados inmediatos

Dimensión 18. Evaluación de resultados intermedios

Dimensión 19. Evaluación de resultados a largo plazo (impacto)

Dimensión 20. Evaluación de resultados posibles pero no planificados

Hay que aclarar que la técnica Delphi no se ha aplicado en su totalidad por las limitaciones de tiempo y de recursos. Por tanto, se realizó una única «vuelta» de los cuestionarios a los expertos por varias razones:

a) El tema ya estaba centrado y no fue necesario realizar cuestionarios con preguntas abiertas o sistemas de grupos nominales para detectar la problemática existente;

b) Se realizó una selección de expertos que permitiera abarcar todo el abanico de puntos de vista posibles. 


\section{TABLA 6: Indicadores de la Dimensión 5 «Diseño del Programa Comprensivo de Orientación (PCO)».}

Indicador 5.1: El PCO se basa en una evaluación del contexto, donde se identifican y analizan las posibilidades, obstáculos, condiciones y limitaciones del entorno en el que se desarrolla el programa.

Indicador 5.2: El PCO se basa en los resultados de la evaluación de necesidades y en los resultados de las evaluaciones anteriores del programa.

Indicador 5.3: El PCO define claramente sus principios, valores y filosofía.

Indicador 5.4: El PCO establece las metas generales a conseguir, basándose en su filosofía, el contexto donde se desarrolla y en las necesidades detectadas.

Indicador 5.5: El PCO establece las competencias específicas que deben conseguir los estudiantes, según las áreas de desarrollo.

Indicador 5.6: El PCO define, operacionaliza y prioriza los objetivos específicos anualmente para lograr las competencias y responder a las necesidades detectadas.

Indicador 5.7: Las competencias específicas y los objetivos reflejan los principios, valores y filosofía del programa, son adecuadas en su desarrollo (según niveles educativos) y son medibles.

Indicador 5.8: El PCO establece una estructura para dirigir las metas del programa y las competencias de los estudiantes:

- Cómo: listas de actividades de acuerdo a cada competencia y objetivo.

- Quién: personal (responsables de cada tarea).

- Con qué: recursos necesarios (presupuesto, infraestructura, materiales y equipamiento).

- Cuándo: tiempo y secuenciación de actividades.

- Para quién: destinatarios (cobertura).

Indicador 5.9: El PCO equilibra adecuadamente todos los componentes y actividades de orientación (desarrollo de competencias, sistema de diagnóstico individual y grupal, orientación individual, orientación de grupo, sistema de asesoramiento comprensivo a estudiantes, actividades de consulta, tareas burocráticas, etc.).

Indicador 5.10: El PCO establece un sistema de valoración global del programa, con criterios y métodos para evaluar su efectividad (la de los objetivos y competencias de los estudiantes).

Indicador 5.11: El PCO se revisa anualmente, según los resultados de la evaluación del programa, nuevas necesidades, cambio de prioridades y nuevos conocimientos de investfigaciones recientes.

Indicador 5.12: El PCO se elabora según los criterios de participación y decisión del personal del centro.

Se realizó a través de un juicio de 18 expertos, distribuidos equilibradamente en tres niveles mediante un muestreo intencionado. Este repertorio de personas se caracteriza por un común denominador: la experiencia y especialización en orientación educativa y en planificación/ evaluación de la orientación. Los criterios que se han utilizado para representar a las posturas que, a priori, podían existir en el tema son:

- Nivel/Perfil: Este criterio ha permitido crear tres grupos con características distintas. Tiene tres categorías: 
a) NiVEL ACADÉMICO-UNIVERSITARIO («teóricos»): proceden del ámbito universitario, aunque anteriormente hayan desempeñado labores como orientadores;

b) NiVEL UNIVERSITARIO-PROFESIONAL («teórico-prácticos»): combinan la enseñanza/investigación en la universidad con el ejercicio de la profesión de orientación, a nivel particular;

c) NiVEL PROFESIONAL («Prácticos»): ejercen de orientadores tanto en departamentos de orientación de colegios e institutos de secundaria (privados y públicos), como en equipos de orientación educativa y profesional. Llevan varios años desempeñando esta profesión.

- Formación:

a) Formación pedagógica;

b) Formación psicológica.

- Tipo de centro:

a) Centros privados;

b) Centros públicos;

c) Centros privados concertados;

d) Universidad;

e) Equipo de orientación educativa y profesional.

- Nivel de acción orientadora:

a) $2^{\circ} \mathrm{Nivel}$ (Departamento de Orientación);

b) $3^{\circ} \mathrm{Nivel}$ (Equipos de Orientación)

- Provincia:

a) Madrid;

b) Toledo;

c) Barcelona;

d) Granada;

e) Santiago de Compostela

Las cuestiones que valoraron los expertos fueron las siguientes:

- Claridad: Valora si el enunciado del indicador es claro, concreto y suficientemente explicativo de la situación que enuncia. Requiere el análisis del contenido del indicador. Su escala de respuesta es dicotómica: Sí/No. Además tiene un apartado para, en su caso, mejorar su enunciado.

- Ponderación y Aceptación: Indica el peso que el experto da a cada indicador en la dimensión a la que pertenece y en función de la importancia para determinar la calidad del programa o para dar información sobre él. Su escala de respuesta es descriptiva (5-1): 5 Imprescindible, 4 Importante, 3 Opcional, 2 Complementario, 1 No se acepta.

- Medida: Señala el tipo de medida más adecuado para cada indicador. Su escala de respuesta es: $\mathrm{CN}=$ Cuantitativa, $\mathrm{CL}=$ Cualitativa, $\mathrm{MT}=$ Mixta.

Para delimitar ese «grado de validez» se definieron una serie de criterios: 
- La suficiencia de las cuestiones incluidas en el S.I.: El S.I. consta de 100 indicadores. El proceso de validación extrajo los indicadores que los expertos consideraban obligatorios. El resto de indicadores formarían la parte opcional. Se obtuvo a través del criterio de ponderación, de forma que todos aquellos indicadores que obtuvieran $>75 \%$ de la respuesta 5 , serían considerados obligatorios.

- La representatividad de la muestra de cuestiones incluidas en el S.I.: Que cada indicador mida un contenido único y diferente; y que cada indicador aporte información relevante. Esto se hizo mediante el criterio de ponderación (escala 1-5), de forma que serían indicadores significativos aquellos que obtuvieran respuestas entre 3-5, y serían indicadores no significativos aquellos que obtuvieran respuestas entre 1-2.

- La especificidad de los indicadores, sin solapamientos entre sí.

- La formulación correcta de los indicadores

- La idoneidad o capacidad de los indicadores para ser evaluados

\section{Resultados}

Se analizaron los datos mediante una estadística descriptiva univariable, que nos permitiera ver la variabilidad de la distribución de los datos, y conocer la amplitud y la homogeneidad/heterogeneidad de los grupos. Para ello, se obtuvieron una distribución de frecuencias y porcentajes, medidas de tendencia central y medidas de dispersión. Además se realizó un análisis de conglomerados que nos permitiera ver la congruencia, agrupamiento y relación de las respuestas que ofrecen los distintos expertos a los tres criterios del cuestionario, de forma que pudiéramos inferir el nivel de robustez del sistema de indicadores. Se muestran a continuación algunos gráficos que describen los porcentajes y promedios de respuesta de los expertos, según las tres cuestiones: claridad, ponderación y medida.

\section{Claridad}

Según el gráfico 3, la dimensión que se juzga como más clara y explicativa (con el $100 \%$ de acuerdo entre los expertos) es la dimensión 7 «CUALIFICACIÓN INICIAL DEL PERSONAL». La dimensión peor valorada es la 2 «APOYO POLÍTICO Y LEGAL AL PROGRAMA COMPRENSIVO DE ORIENTACIÓN» con un $20{ }^{\prime} 59 \%$ de desacuerdo. A pesar de la buena valoración que han tenido la práctica totalidad de las dimensiones del sistema (obtenido según el promedio de los indicadores que las componen), es necesario señalar que cinco dimensiones $(16,17$, 18,19 y 20) no han sido contestadas por un experto. Sin embargo, no se ofrecieron modificaciones a estos indicadores poco claros. Esto nos hace pensar en los efectos que pudo tener la longitud del protocolo de indicadores y el cansancio que pudo causar, ya que estas dimensiones están situadas justo al final del protocolo.

En cuanto al grupo de expertos, el grupo de «prácticos» considera que el S.I. es bastante claro y preciso (con un 93\%) y los que menos claro lo perciben son el grupo de «teóricos» (con un 89'50\%). Además, este grupo es el que obtiene mayor porcentaje de respuestas en blanco (sin respuesta) (un 2\%). 


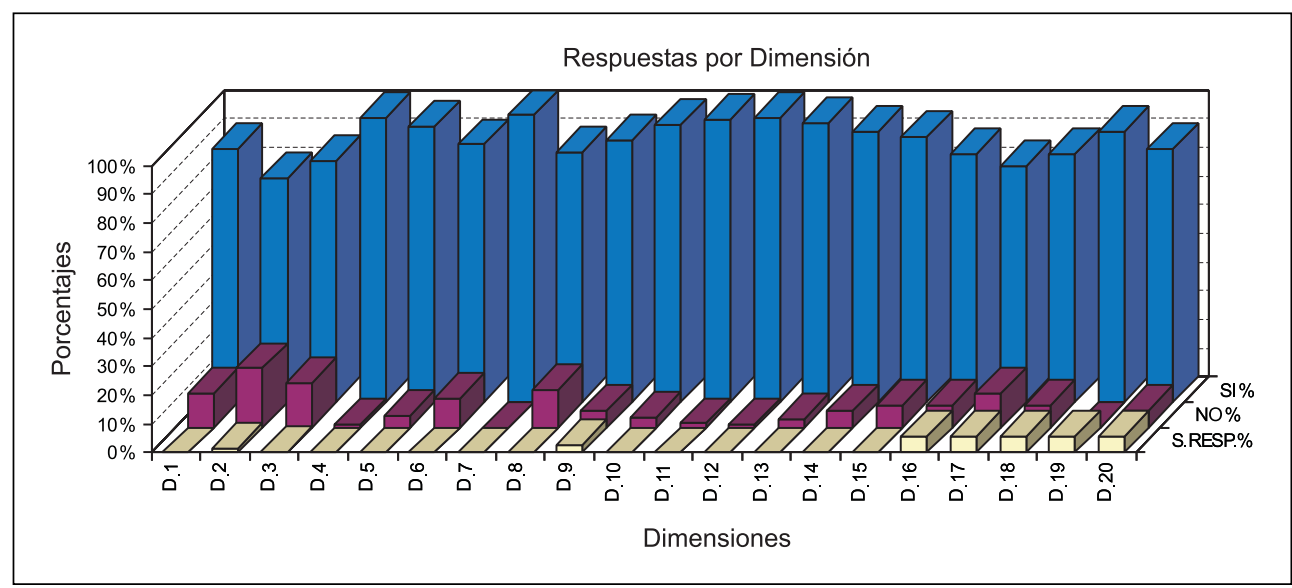

\section{GRÁFICO 3. \\ Porcentajes de respuesta por dimensión (Claridad).}

Resumiendo, es de destacar la homogeneidad de las respuestas, tanto a lo largo de los indicadores como de los expertos. Parece que, independientemente de lo mejorable de los indicadores, los expertos pertenecientes al ámbito universitario hicieron más hincapié en el aspecto formal del enunciado y el grupo de «prácticos» prestó más atención al nivel de comprensión que tenía cada indicador, no fijándose tanto en el aspecto formal y gramatical.

\section{Ponderación}

En cuanto al criterio de ponderación, en la tabla 7 y gráfico 4 , se muestran los porcentajes y medidas promedio y de dispersión correspondientes a las dimensiones. Apuntamos a continuación, algunos de los resultados. La totalidad de las dimensiones se consideran Imprescindibles o Importantes. Las dimensiones que obtienen mayor consenso en torno al peso 5 (Imprescindible) son las D.5 «DISEÑO DEL PCO» con un 55’39\% y D.13 «POLÍTICA/LEGISLACIÓN MINISTERIAL, AUTONÓMICA, LOCAL Y DEL CENTRO» con un 55'88\%. La que obtiene mayor consenso en torno al peso 4 (Importante) es la dimensión D1 «CARACTERÍsTICAS DEL ENTORNO» con un 55'88\%. Resumiendo, se observa una buena valoración general de las dimensiones, una alta participación de todos los expertos y un alto consenso y afinidad en las respuestas en torno al peso 5 (39'71\%) y al peso $4\left(38^{\prime} 18 \%\right)$.

En cuanto a los promedios de respuesta, las dimensiones que obtienen mejor valoración y mayor homogeneidad en sus respuestas son, por orden de importancia, la D.5, D.13, D.2, D.4, D.7, D.3, D.12, D.15, D.1. El resto de dimensiones obtienen promedios en torno al peso 3 (Opcional). Las dimensiones menos valoradas son la D.19 «EVALUACIÓN DE RESULTADOS A LARGO PLAZO» y la D.20 «EVALUACIÓN DE RESULTADOS POSIBLES PERO NO PLANIFICADOS».

El peso que obtiene mayor consenso es el 5 y el 4 . Aunque, a nosotros nos interesa el peso que obtiene cada indicador en particular, el hecho de realizar promedios de las dimen- 


\section{TABLA 7: Porcentajes de respuesta, media, desviación típica y varianza por dimensión (Ponderación).}

\begin{tabular}{|c|c|c|c|c|c|c|c|c|c|}
\hline \multirow{2}{*}{$\begin{array}{c}\mathrm{N}^{0} \\
\text { Dimensión }\end{array}$} & \multicolumn{4}{|c|}{ Aceptación (\%) } & \multirow{2}{*}{$\frac{\operatorname{Rechazo}(\%)}{1}$} & \multirow{2}{*}{$\begin{array}{c}\text { Sin respuesta } \\
(\%)\end{array}$} & \multirow{2}{*}{$\overline{\mathbf{x}}$} & \multirow{2}{*}{$\mathbf{s}$} & \multirow{2}{*}{$s^{2}$} \\
\hline & 5 & 4 & 3 & 2 & & & & & \\
\hline D.1 & $29,41 \%$ & $55,88 \%$ & $8,82 \%$ & $2,94 \%$ & $0,00 \%$ & $2,94 \%$ & 4,15 & 0,71 & 0,51 \\
\hline D. 2 & $42,65 \%$ & $36,76 \%$ & $11,76 \%$ & $1,47 \%$ & $0,00 \%$ & $7,35 \%$ & 4,33 & 0,75 & 0,57 \\
\hline D. 3 & $40,72 \%$ & $38,91 \%$ & $8,60 \%$ & $3,62 \%$ & $1,36 \%$ & $6,79 \%$ & 4,21 & 0,88 & 0,77 \\
\hline D. 4 & $41,18 \%$ & $47,06 \%$ & $7,06 \%$ & $2,35 \%$ & $1,18 \%$ & $1,18 \%$ & 4,27 & 0,79 & 0,63 \\
\hline D. 5 & $55,39 \%$ & $33,82 \%$ & $8,82 \%$ & $0,98 \%$ & $0,00 \%$ & $0,98 \%$ & 4,45 & 0,70 & 0,49 \\
\hline D.6 & $30,88 \%$ & $38,24 \%$ & $13,24 \%$ & $7,35 \%$ & $2,94 \%$ & $7,35 \%$ & 3,95 & 1,05 & 1,09 \\
\hline D.7 & $39,71 \%$ & $48,53 \%$ & $5,88 \%$ & $5,88 \%$ & $0,00 \%$ & $0,00 \%$ & 4,22 & 0,81 & 0,65 \\
\hline D.8 & $32,94 \%$ & $40,00 \%$ & $9,41 \%$ & $3,53 \%$ & $3,53 \%$ & $10,59 \%$ & 3,92 & 1,00 & 1,00 \\
\hline D.9 & $27,94 \%$ & $36,76 \%$ & $23,53 \%$ & $2,94 \%$ & $1,47 \%$ & $7,35 \%$ & 3,72 & 0,91 & 0,83 \\
\hline D. 10 & $35,29 \%$ & $36,47 \%$ & $9,41 \%$ & $8,24 \%$ & $9,41 \%$ & $1,18 \%$ & 3,80 & 1,28 & 1,63 \\
\hline D. 11 & $41,18 \%$ & $27,94 \%$ & $16,18 \%$ & $8,82 \%$ & $5,88 \%$ & $0,00 \%$ & 3,90 & 1,21 & 1,47 \\
\hline D. 12 & $38,24 \%$ & $44,12 \%$ & $13,73 \%$ & $2,94 \%$ & $0,00 \%$ & $0,98 \%$ & 4,19 & 0,78 & 0,61 \\
\hline D.13 & $55,88 \%$ & $35,29 \%$ & $5,88 \%$ & $0,00 \%$ & $2,94 \%$ & $0,00 \%$ & 4,41 & 0,86 & 0,73 \\
\hline D. 14 & $35,29 \%$ & $32,35 \%$ & $20,59 \%$ & $0,00 \%$ & $11,76 \%$ & $0,00 \%$ & 3,79 & 1,27 & 1,62 \\
\hline D. 15 & $43,44 \%$ & $34,84 \%$ & $12,67 \%$ & $2,26 \%$ & $2,26 \%$ & $4,52 \%$ & 4,17 & 0,93 & 0,86 \\
\hline D.16 & $37,82 \%$ & $34,45 \%$ & $14,29 \%$ & $2,52 \%$ & $0,84 \%$ & $10,08 \%$ & 3,94 & 0,87 & 0,75 \\
\hline D. 17 & $31,37 \%$ & $33,33 \%$ & $15,69 \%$ & $7,84 \%$ & $0,00 \%$ & $11,76 \%$ & 3,75 & 0,95 & 0,91 \\
\hline D. 18 & $25,49 \%$ & $47,06 \%$ & $7,84 \%$ & $5,88 \%$ & $3,92 \%$ & $9,80 \%$ & 3,66 & 1,02 & 1,04 \\
\hline D. 19 & $17,65 \%$ & $41,18 \%$ & $5,88 \%$ & $11,76 \%$ & $11,76 \%$ & $11,76 \%$ & 3,06 & 1,36 & 1,84 \\
\hline D. 20 & $11,76 \%$ & $47,06 \%$ & $23,53 \%$ & $0,00 \%$ & $5,88 \%$ & $11,76 \%$ & 3,24 & 0,98 & 0,95 \\
\hline TOTAL & $39,71 \%$ & $38,18 \%$ & $11,47 \%$ & $3,59 \%$ & $2,24 \%$ & $4,82 \%$ & & & \\
\hline
\end{tabular}

siones y factores obedece al interés en conocer qué peso o importancia predomina más para los contenidos generales que define cada dimensión y cada indicador.

En cuanto a los porcentajes de respuesta por factor (gráfico 5), el factor Diseño del programa obtiene el grado de homogeneidad más alto en sus respuestas. A pesar de la magnitud de las desviaciones, las medias obtenidas confirman que existe un consenso generalizado en valorar los indicadores como Imprescindibles e Importantes. Los factores que obtienen mejor valoración y mayor homogeneidad en sus respuestas son, por orden de importancia, el factor Diseño, el factor Contexto, el factor Aplicación, el factor Evaluación, el factor Recursos y el factor Resultados.

En cuanto al grupo de expertos, existe un patrón de respuesta similar en los tres grupos de expertos y para los 6 factores, excepto el factor de Aplicación, en que el grupo de Teóri- 


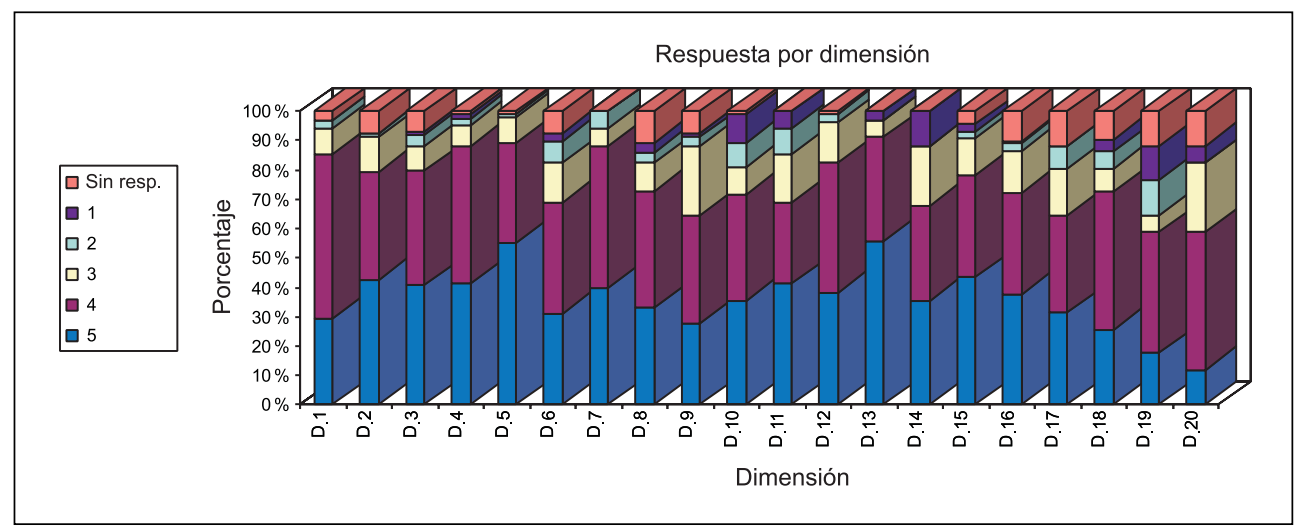

\section{GRÁFICO 4. \\ Porcentajes de respuesta por dimensión (Ponderación).}

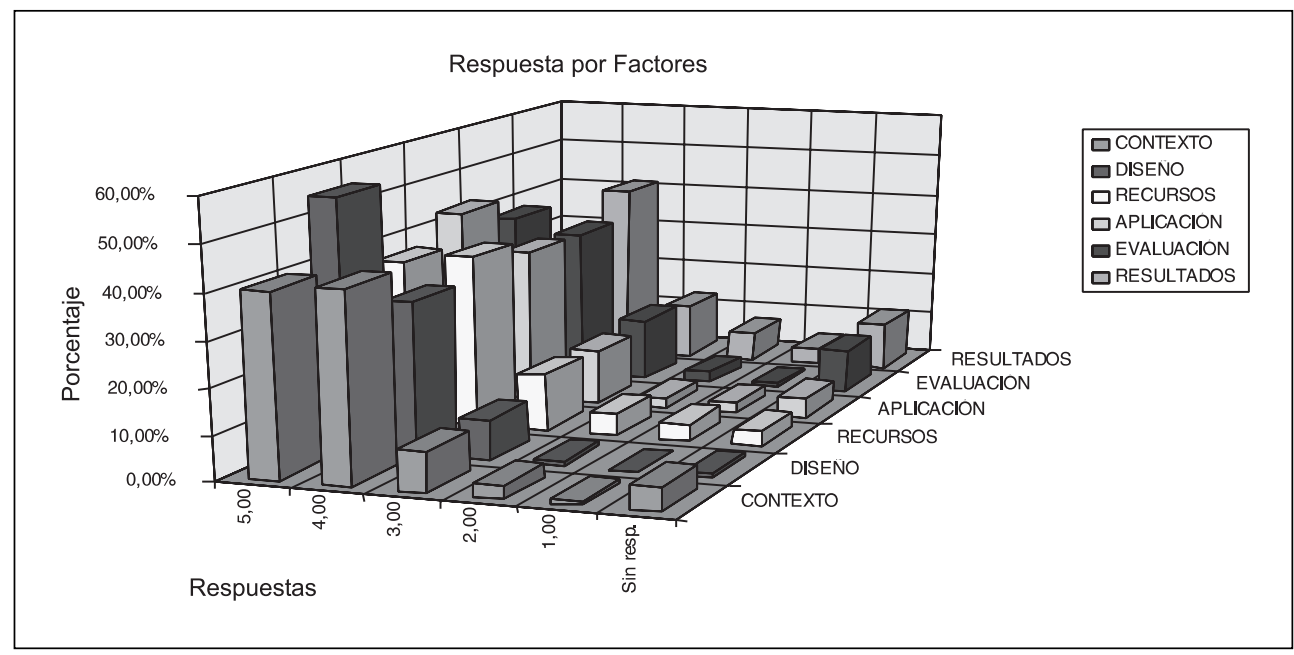

\section{GRÁFICO 5. \\ Porcentajes de respuesta por factor (Ponderación).}

cos lo valora por debajo de 4 (Importante). Los factores más valorados son Diseño, Aplicación y Contexto; y los menos valorados son Evaluación, Recursos y Resultados, por orden decreciente (gráfico 6).

Estos resultados reflejan la situación y necesidades actuales de la orientación educativa en los centros, pues estamos en un momento de consolidación de la orientación. Es difícil plantearse el tema de la Planificación de la Evaluación y los Resultados, cuando falta todavía la dinámica de trabajar sistemáticamente por programas. En la perspectiva de la evaluación comprensiva, se pretende realizar ambas (planificación y evaluación) simultáneamente. 


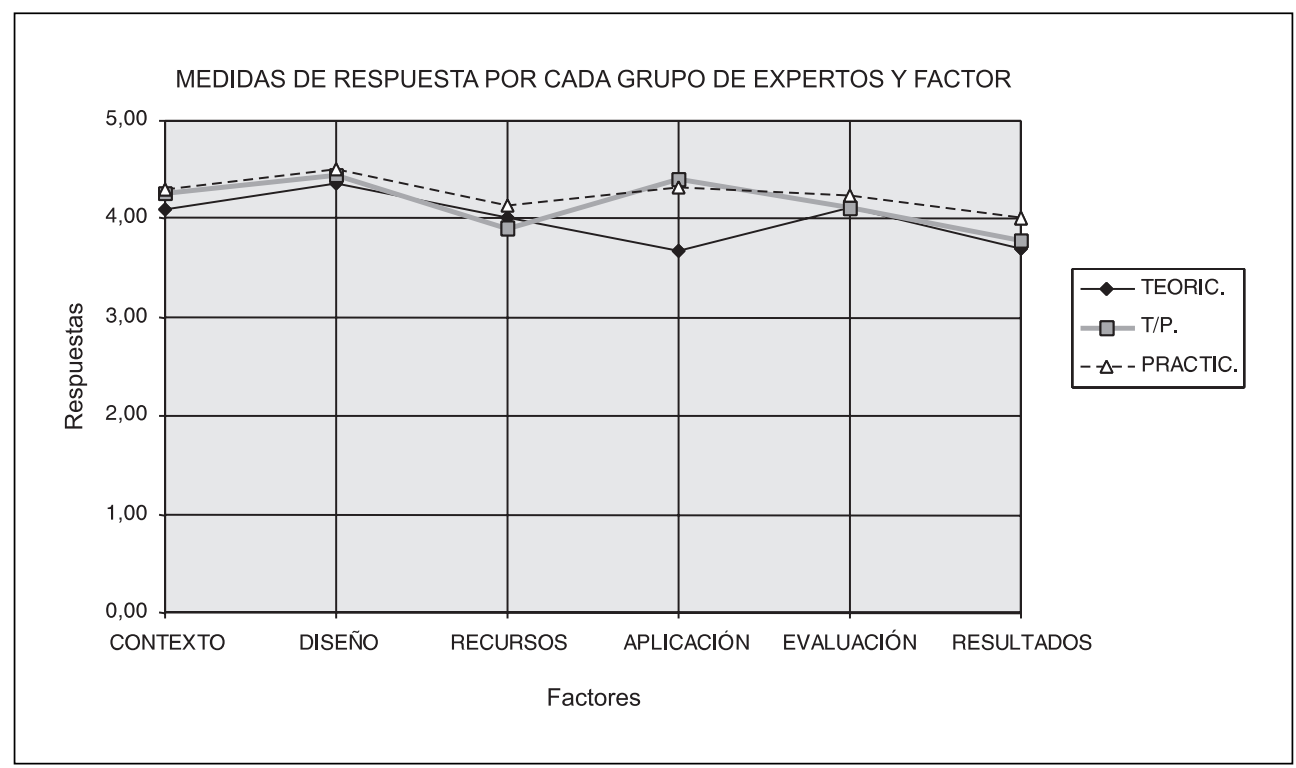

GRÁFICO 6.
Promedios de respuesta por cada grupo de expertos y factor (Ponderación).

\section{Medida}

En términos globales y en orden decreciente, se obtiene un $41{ }^{\prime} 18 \%$ a favor de la medida cualitativa, un $40,18 \%$ favorables a una medida mixta y un $12{ }^{\prime} 47 \%$ para la medida cuantitativa. Por tanto, parece que la medida que predomina más es la medida cualitativa y la mixta. Puede decirse que hay un patrón de conducta en el tipo de respuestas dadas.

Las dimensiones que obtienen mayor consenso son las D.2, D.3, D.4, D.7, D.13 y D.14 con una medida cualitativa, la D.17, D.18, D.19 y D.20 con una medida mixta y, por último, la D.10 con una medida claramente cuantitativa. El resto de dimensiones tienen un grado de dispersión alto, por lo que no puede deducirse cuál es el tipo de medida más adecuado (ver gráfico 7).

En cuanto a los grupos de expertos, la gran mayoría se decantan por una medida cualitativa o mixta. Además, independientemente de la medida particular que obtiene cada indicador, no existen diferencias significativas en el tipo de medida que obtiene cada grupo de expertos (gráfico 8).

\section{Análisis cluster}

Se realizaron análisis de conglomerados por sujetos. Sin embargo, no se han encontrado grupos con patrones de respuesta similares, ni tampoco parece coincidir la variabilidad de las respuestas según el perfil de los tres grupos de expertos que se definieron inicialmente. 


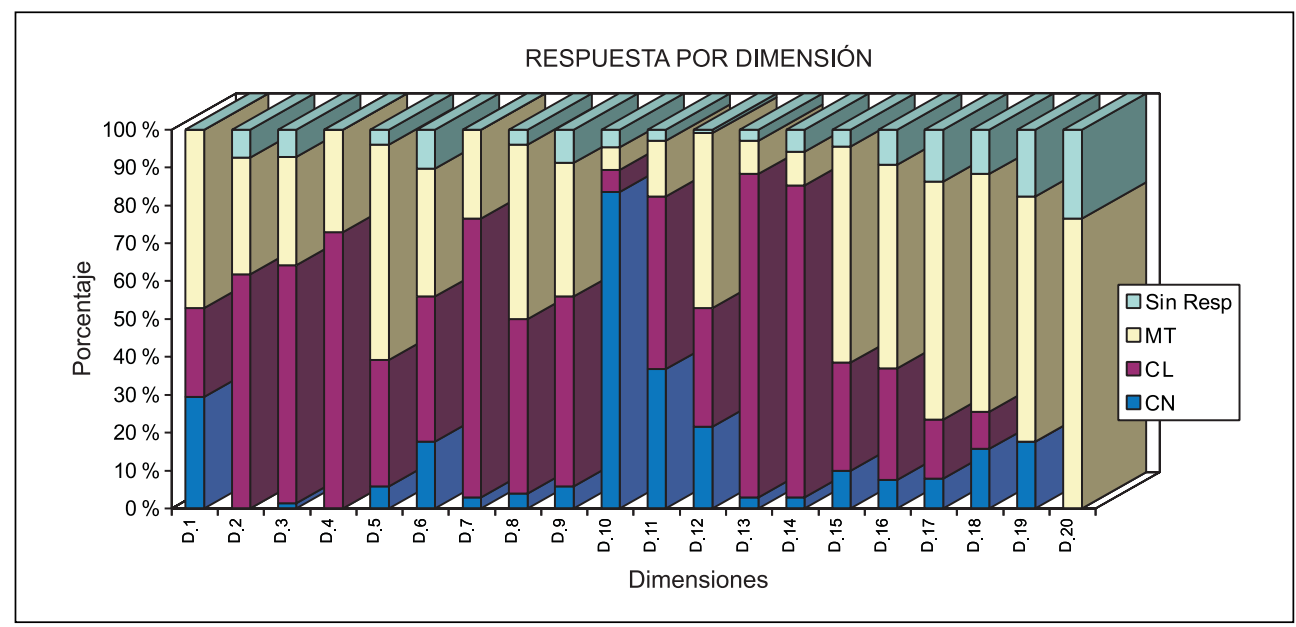

GRÁFICO 7.

Porcentajes de respuesta por dimensión (Medida).

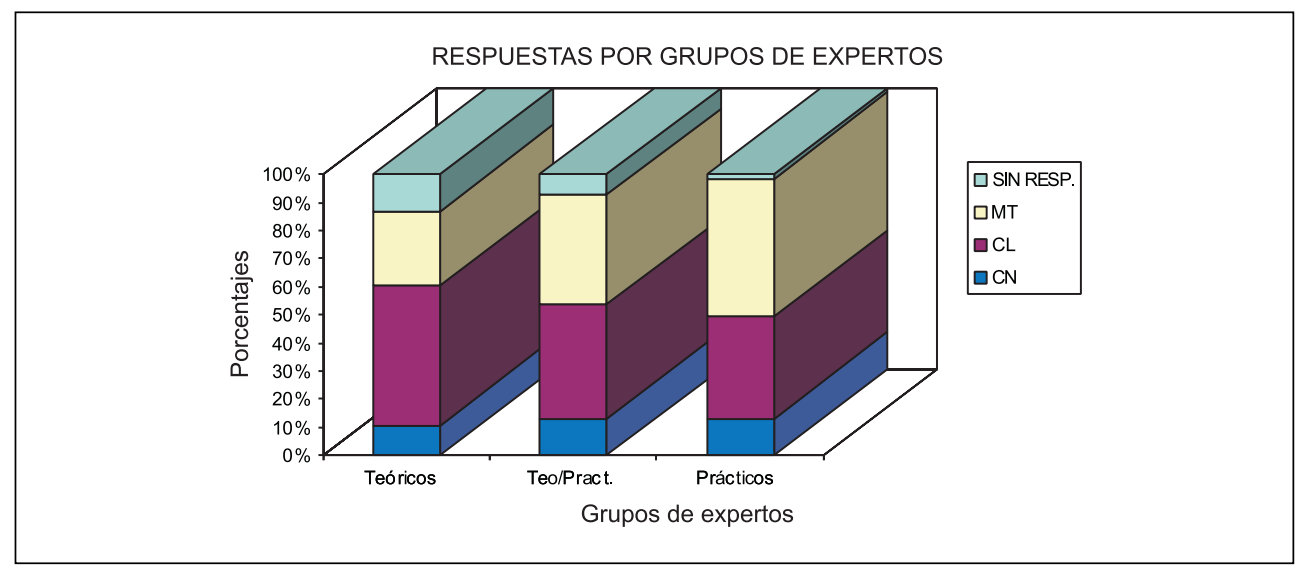

GRÁFICO 8.

Porcentajes de respuesta por grupos de expertos (Medida).

\section{Conclusiones}

Según los resultados obtenidos, se llegó a las siguientes conclusiones:

\section{Criterio de Claridad}

a) El grupo de teóricos señala un nivel de dificultad mayor que el grupo de teórico/prácticos y el grupo de prácticos; 
b) Los indicadores que resultan más claros son Diseño y Ejecución del programa y los que resultan menos claros son Planificación de la evaluación y Resultados;

c) Existe una similitud de respuestas a lo largo de todos los indicadores de las dimensiones entre los expertos.

De cualquier forma, se mejoró la formulación de los indicadores, haciéndolos más sencillos y precisos, especialmente en la D2. Apoyo legal y político al PCO, D3. Estructura del PCO en el centro, y D9. Competencia y trabajo del orientador.

\section{Criterio de Aceptación y Ponderación}

a) Existe una similitud en la respuesta a lo largo de todos los indicadores. La mayor parte se consideran Imprescindibles (5) e Importantes (4). Alguno de ellos se considera Opcional (3);

b) La dimensión 3 «ESTRUCTURA DEL PROGRAMA Y ORGANIZACIÓN DEL PERSONAL» ha sido la más problemática y confusa para los expertos, precisamente porque trata de la organización de la orientación, tema algo confuso actualmente y polisémico por la doble vertiente que existe de servicios/programas;

c) Los factores mejor valorados han sido, por orden de prioridad, el factor Diseño, Contexto, Aplicación, Evaluación, Recursos y Resultados. Es significativo el interés que despierta el factor Diseño del programa, a diferencia del factor Resultados, menos valorado;

d) En general, existe un consenso sustancial en el grado de importancia dado a los indicadores del sistema;

e) Los que mejor valoran los indicadores son el grupo de «teórico-prácticos» a diferencia del grupo de teóricos, que lo valora con menos intensidad.

En síntesis, de los 100 indicadores posibles, sólo 16 se han considerado obligatorios (Imprescindibles) (tabla 8). Los demás serán opcionales para cada EOEP. Como vemos, todos pertenecen a aspectos de entrada que habitualmente son parte del trabajo diario del orientador. Sin embargo, no se consideran Imprescindibles, aunque sí Importantes, los indicadores relativos a Aplicación del programa, Evaluación y Resultados. Parece apreciarse una tendencia a considerar Imprescindible aquellos aspectos con los que estamos acostumbrados a trabajar o a los elementos que nos vienen dados desde fuera. Según nuestra opinión, la conciencia de la necesidad de algo quizá pueda darse con mayores cotas de autonomía que las actuales, donde en ocasiones el trabajo orientador se mira más desde la perspectiva de la normativa que desde la perspectiva de la calidad de la orientación y de su eficacia.

De cualquier forma, de todo el abanico de indicadores propuesto, se corrigieron algunos solapamientos existentes entre indicadores, especialmente en algunas dimensiones como la D3. Organización del programa y D9. Competencia y trabajo del orientador; y se ubicaron mejor algunos indicadores, como los pertenecientes a la D4. Organización y mejora del personal. Además, prácticamente la totalidad de los indicadores han resultado significativos porque obtuvieron unos porcentajes de respuesta entre 3-5 (opcional y imprescindible). Sin embargo, los indicadores de la D.10 Presupuesto es la que recibe más porcentaje de recha- 
TABLA 8: Indicadores obligatorios.

\begin{tabular}{|l|l|l|}
\hline FACTORES & \multicolumn{1}{|c|}{ DIMENSIONES } & \multicolumn{1}{c|}{ INDICADORES } \\
\hline $\begin{array}{l}\text { Factor I. } \\
\text { Contexto }\end{array}$ & D3. Organización del PCO & $\begin{array}{l}\text { 3.4. Constitución del Dpto. o. EOEP } \\
\text { 3.5. Coordinación del PCO }\end{array}$ \\
\hline $\begin{array}{l}\text { Factor II. } \\
\text { Diseño del } \\
\text { programa }\end{array}$ & D6. Diseño y elaboración del PCO & $\begin{array}{l}\text { 6.8. Actividades } \\
\text { 6.10. Personal } \\
\text { 6.11. Recursos necesarios } \\
\text { 6.12. Tiempo y secuenciación de } \\
\text { actividades }\end{array}$ \\
\hline $\begin{array}{l}\text { Factor III. } \\
\text { Recursos }\end{array}$ & D7. Disponibilidad de personal & $\begin{array}{l}\text { 6.13. Destinatarios } \\
\text { 6.15. Valoración del PCO }\end{array}$ \\
\hline & D8. Cualificación del personal & 8.1. Formación inicial del personal \\
\hline & D9. Competencia y trabajo del & $\begin{array}{l}\text { 9.1. Funciones y roles profesionales } \\
\text { orientador }\end{array}$ \\
& & $\begin{array}{l}\text { 9.2. Responsabilidades del orientador y } \\
\text { personal de apoyo }\end{array}$ \\
& & $\begin{array}{l}\text { 9.3. Competencias necesarias (habilidad, } \\
\text { conoc, personalidad) }\end{array}$ \\
& D12. Infraestructuras & $\begin{array}{l}\text { 12.1. Adecuación y accesibilidad de } \\
\text { espacios }\end{array}$ \\
\hline & D13. Materiales y equipamiento & $\begin{array}{l}\text { 13.2. Materiales de diagnóstico (tests, } \\
\text { escalas, pruebas manipulativas, etc) }\end{array}$ \\
\hline & &
\end{tabular}

zos, especialmente el indicador 10.5 Presupuesto asignado para mantenimiento, precisamente porque son partidas que se organizan y gestionan desde fuera.

\section{Criterio de Medida}

Aunque lo que interesa es la medida particular de cada indicador, en general, la medida más frecuente para casi todos los indicadores ha sido la cualitativa y la mixta, excepto la D11. Presupuesto, que es claramente cuantitativa.

El objetivo era elaborar un instrumento para evaluar los efectos de la orientación educativa. Para ello, es necesario disponer de un programa de orientación. Sin embargo, actualmente no todas las tareas de orientación se rigen por un programa, o no todos los «programas» tienen las características técnicas que lo definen. Por ello, deben iniciarse sistemas de evaluación sencillos y breves hasta ir trabajando sistemáticamente por programas.

Según los resultados de la validación, se obtuvo un segundo borrador del S.I. acorde a las sugerencias de los expertos, mejorándose la formulación, eliminando solapamientos de 
indicadores, ubicando mejor otros y asignando un peso a cada indicador. Las próximas tareas son:

a) contextualizar los indicadores en los D.O. y Equipos de Orientación a través de una pauta que ya estamos iniciando, para elaborar una aproximación entre las relaciones y correspondencias entre indicadores;

b) Elaborar unas Guías de recogida de información para cada indicador.

Los indicadores permiten no sólo evaluar los efectos sino ver las tendencias de determinadas acciones y ayudar, así, a planificar mejor. Algunas de las aplicaciones de los indicadores al evaluar la orientación educativa son:

\section{En procesos/actividades}

- Disponibilidad de un sistema de evaluación contextualizado: adecuación del sistema de indicadores al problema que tenemos, de manera que la evaluación interna que planificamos pueda ser flexible para responder mejor a las áreas que nos interesan.

- Mayor profundización y veracidad de los datos: permitirá analizar el carácter cualitativo de lo realizado para aportar fortaleza en la interpretación de los datos.

- Evidenciar qué servicios personales y recursos materiales conviene incrementar.

- Estimación de la rentabilidad económica y psicopedagógica: recibir una constante información sobre el grado de efectividad del programa.

\section{Impacto social}

- Aceptación de la autoevaluación: la realización de la evaluación por toda la comunidad implicada en el proceso de orientación permite crear una cultura de evaluación dentro de los Equipos Psicopedagógicos y D.O., evitando el recelo y rechazo que produce toda acción evaluadora.

- Estilo de trabajo: facilita una organización del trabajo más operativa y mayor coordinación, ya que el proceso se programa según los datos objetivos y puntuales que va ofreciendo la evaluación.

- Rentabilidad a nivel de conductas: el trabajo del personal implicado es más efectivo por el feedback recibido en la autoevaluación.

- Perfeccionamiento profesional de los orientadores: la detección de lagunas formativas y deficiencias en el trabajo permitirá analizar las necesidades de los orientadores para cubrirlas según un orden de prioridad.

\section{Impacto educativo}

- Poder identificar a los estudiantes cuyas necesidades prioritarias no están siendo satisfechas, así como detectar las necesidades reales de orientación de alumnos, profesores y padres. 
- Llevar un seguimiento de los efectos de la intervención a lo largo del tiempo, para obtener información parcial del proceso y variar o no dicha intervención.

- Elegir aquellas técnicas que ofrecen mayor probabilidad de eficacia y justificar la eliminación de las actividades que no resulten útiles.

\section{Bibliografía}

Alonso TAPIA, J. (1995): Diseño y evaluación de programas de orientación educativa. En: Orientación educativa. Teoría, evaluación e intervención. Madrid: Síntesis-Psicología.

Álvarez Rojo, V. (1987): Diseño de programas de orientación educativa. En: V. Álvarez Rojo (Ed): Metodología de la orientación educativa. Sevilla: Morata.

Anguera Arguilaga, M. T. (1990): Programas de intervención. ¿Hasta qué punto es factible su evaluación? Revista de investigación educativa, 8, 16, 77-94.

Anguera Arguilaga, M. T. (1991): Evaluación de programas en servicios sociales: alternativas metodológicas. Madrid: CICYT.

AtKinson, D. R.; Furlong, M. J. y Janoff, D. S. (1979): A four-component model for proactive accountability in school counseling. School counselor, March, 26, 222-228.

Borders, L. D. y DRURY, S. M. (1992a): Comprehensive school counseling programs: a review for policymakers and practitioners. Journal of counseling and development. March/April, 70, 487498.

Borders, L. D. y DruRY, S. M. (1992b): Counseling programs: a guide to evaluation. Newbury Park, London: Corwin Press.

Daniels, M. H.; Mines, R. y Gressard, C. (1981): A meta-model for evaluating counseling programs. Personnel and guidance journal, May, 59, 578-582.

FAirchild, T. N. y SEeley, T. J. (1995): Accountability strategies for school counselors: a Baker's Dozen. School counselor. May, 42, 377-392.

Frith, G. H. y ClaRK, R. (1982): Evaluating elementary school counseling programs: 10 common myths of practitioners. Elementary school guidance and counseling, 17, 49-51.

Gysbers, N. C. y Henderson, P. (1994): Developing and managing your school guidance program ( $2^{\mathrm{a}}$ ed). Alexandria, VA: ACA.

Gysbers, N. C. y Henderson, P. (1997): Comprehensive guidance programs that work-II. Greensboro, NC: ERIC/CASS.

Henderson, P. (1994a): Administrative skills in counseling supervision. En: L. D. Borders (Ed.) Supervision: Exploring the effective components. Greensboro, NC: ERIC/CASS.

Henderson, P. (1994b): Guidance and counseling have a new look in today's secondary schools. Texas study of secondary education, III (3), 38-39.

Henderson, P. (1994c): Supervision of school counselors. En: L. D. Borders (Ed.) Supervision: Exploring the effective components. Greensboro, NC: ERIC/CASS.

Henderson, P. y GysBers, N. C. (1998): Leading and managing your school guidance program staff. ACA, Alexandria, VA.

Henderson, P.; Bailey, M.; Krueger, D. y Williams, L. (1996): A visit to a comprehensive guidance program at work. Greensboro, NC: ERIC/CASS. 
Hernández Fernández, J. y Martínez Clares, P. (1996): Propuesta metodológica para evaluar programas de orientación educativa. Revista electrónica de investigación y evaluación educativa, vol. $2, n^{\circ} 2-3$.

Hughey, K. F.; Gysbers, N. C. y Starr, M. (1993): Evaluating comprehensive school guidance programs: assessing the perceptions of students, parents and teachers. School counselor, 41, 31-35.

Johnson, S. K. y Whitfield, E. A. (Eds.) (1991): Evaluating guidance programs: A practicioner's guide. Iowa City: American college testing publications.

Krumboltz, J. D. (1974): An accountability model for counselors. Personnel and guidance journal, $52,639-646$

LÁzaro Martínez, A. (1991): La formalización de indicadores de evaluación. Bordón, 43, 4, 477494.

Manzano Soto, N. (1997): Evaluación de la intervención orientadora. ICCE, 240, 18-23.

Missouri University (1991a): Guidance Kit. Missouri Comprehensive Guidance: a model for program development, implementation and evaluation. Middle School (6-9). Columbia. Instructional Materials Lab. ED 349487.

Missouri University (1991b): Guidance Kit. Missouri Comprehensive Guidance: a model for program development, implementation and evaluation. Secondary School (9-12). Missouri Univ., Columbia. Instructional Materials Lab. ED 349488.

Montané, J. y MARTínez, M. (1994): La orientación escolar en la educación secundaria. Una nueva perspectiva desde la educación para la carrera profesional. Barcelona: PPU.

NoRTHSIDE INDEPENDENT SCHOOL DisTRICT (1997): Guide to counselor performance improvement through job definition, professionalism assessment, supervision, performance evaluation and professional development. San Antonio, TX: Guidance Department.

OAKES, J. (1986): Educational indicators: a guide for policymakers. New Brunswick, N.J.Rudges University: Center for Policy Research in Education.

PÉREz Juste, R. (1992): Evaluación de programas de orientación. V Seminario Iberoamericano de orientación, Tenerife, 13-16 mayo AEOEP.

Rodríguez Espinar, S. (1988a): La orientación educativa y la calidad de la educción. Bordón, 40 (2), $235-255$.

Rodríguez Espinar, S. (1988b): Calidad de la educación y orientación: implicaciones en el modelo de intervención de los servicios de orietnación. En Actas de las I Jornadas de orientación escolar y profesional. Canarias, AEOEP, 39-54.

RodrígUEZ EsPINAR, S. (1991): Calidad universitaria: un enfoque institucional y multidimensional. En M. DE Miguel y otros: La evaluación de las instituciones universitarias. Madrid, Consejo de Universidades, Secretaría general, 39-72.

Rodríguez Espinar, S. (1995): Un reto profesional: la calidad de la intervención orientadora. En R. SANZ Oro y otros (Eds.): Tutoría y orientación. Barcelona: CEDECS, 119-135.

Rodríguez Espinar, S. (Coord.), Alvarez, M.; Echevarría, B. y Marín, M. A. (1993): Teoría y práctica de la orientación educativa. Barcelona: PPU.

SANDERS, J. R. (1994): The program evaluation standards: how to assess evaluation of educational programs. Thousand Oaks, CA: Sage.

SAnz Oro, R. (1992): Propuesta de planificación y evaluación de un programa comprensivo de orientación educativa. Bordón, 44, 145-152.

Sanz Oro, R. (1996): Evaluación de programas de orientación educativa (2ªed). Madrid: Pirámide. 
SChaffer, J. L. y AtKinson, D. R. (1983): Counselor education courses in program evaluation and scientific research: ares counselors being prepared for the accountability press. Counselor education and supervision, 23, 29-34.

SCHEEREns, J. (1991): Process indicators of school functioning. A selection based on the research literature on school effectiveness. Studies in educational evaluation, 17, 371-404.

SElden, R. W. (1990): Developing educational indicators: A state-national perspective. International Journal of Educational research, 14, 383-393.

Stufflebeam, D. L. y Shinkfield, A. J. (1987): Evaluación sistemática. Madrid: Paidós: MEC.

Tejedor, F. J.; García-Valcárcel, A. y Rodríguez Conde, Mª J. (1994): Perspectivas metodológicas actuales de la evaluación de programas. Revista de investigación educativa, 23, 93-128.

Texas Education Agency (1996): Texas school counseling and guidance programs. Final study report. Austin, TX: Author.

The Scottish Office, Education Department (1992): Using Performance Indicators in Secondary School Self-Evaluation. HM Inspectors for Schools. School Development Planning Support Materials.

Fecha de recepción: 20-3-00

Fecha de revisión: 5-4-00

Fecha de aceptación: 11-4-00 